\title{
Effective regression testing for model-based automotive software using stochastic error propagation analysis
}

Andrey Morozov, T. Chen, K. Ding, K. Janschek

IFA, TU Dresden

This manuscript is not available according to publishing restriction. Thank you for your understanding.

(C) Springer Fachmedien Wiesbaden GmbH 2017

M. Bargende, H.-C. Reuss, J. Wiedemann (Hrsg.), 17. Internationales Stuttgarter Symposium, Proceedings, DOI 10.1007/978-3-658-16988-6_57 